\title{
Network State Classification based on THE STATISTICAL PROPERTIES OF RTT FOR AN Adaptive Multi-State Proactive Transport PROTOCOL FOR SATELLITE BASED NETWORKS
}

\author{
Mohanchur Sakar $^{1}$, K.K.Shukla ${ }^{2}$ and K.S.Dasgupta ${ }^{2}$ \\ ${ }^{1}$ Space Applications Centre (ISRO), Ahmedabad, Gujarat, India \\ mohanchureyahoo.com/msarkaresac.isro.gov.in,ksdesac.isro.gov.in \\ ${ }^{2}$ Department of Computer Engineering, I.T, BHU, Varanasi, U.P, India \\ kkshukla.cseeitbhu.ac.in
}

\begin{abstract}
This paper attempts to establish the fact that Multi State Network Classification is essential for performance enhancement of Transport protocols over Satellite based Networks. A model to classify Multi State network condition taking into consideration both congestion and channel error is evolved. In order to arrive at such a model an analysis of the impact of congestion and channel error on RTT values has been carried out using ns2. The analysis results are also reported in the paper. The inference drawn from this analysis is used to develop a novel statistical RTT based model for multi state network classification. An Adaptive Multi State Proactive Transport Protocol consisting of Proactive Slow Start, State based Error Recovery, Timeout Action and Proactive Reduction is proposed which uses the multi state network state classification model. This paper also confirms through detail simulation and analysis that a prior knowledge about the overall characteristics of the network helps in enhancing the performance of the protocol over satellite channel which is significantly affected due to channel noise and congestion. The necessary augmentation of ns 2 simulator is done for simulating the multi state network classification logic. This simulation has been used in detail evaluation of the protocol under varied levels of congestion and channel noise. The performance enhancement of this protocol with reference to established protocols namely TCP SACK and Vegas has been discussed. The results as discussed in this paper clearly reveal that the proposed protocol always outperforms its peers and show a significant improvement in very high error conditions as envisaged in the design of the protocol. The protocol is also evaluated to have very good fairness property under different error conditions.
\end{abstract}

\section{KEYWORDS}

GEO, ns2, Proactive TCP, SACK, Vegas, Adaptive

\section{INTRODUCTION}

TCP has become the de-facto protocol standard for congestion control in the existing terrestrial Internet. However, experimental and analytical studies [13] confirm that the current TCP protocol variants have performance problems in long fat networks coupled with very high wireless channel errors like satellite networks [16], [15], [21]. Satellite based Networks are dominated by random packet errors which are not common in the wired counterpart. TCP protocols react to the lack of arrival of acknowledgments or duplicate ACK as a sign of congestion. So the congestion window is reduced which leads to unnecessary throughput degradation [13]. It is a challenge for the network researchers and protocol developers to find means to differentiate the cause of the DUP ACK arrival. Generally probing is done in protocols like Peach [1], Peach+ [2], TP-Planet [3] and RCS [4] but at the cost of bandwidth used for the low priority dummy packets. 
Majority of the Transport Protocols tries to ascertain the condition of the network in terms of an estimation of the prevailing congestion in the network [8]. This is because of the fact that the basic paradigm on which the conventional TCP protocols evolved was based on a wired connectivity where congestion was the primary concern. The channel noise was not taken into account mainly due to the fact that wired connectivity can be through of as an errorless channel [13]. So the network states were broadly classified into two states congested and uncongested. But when TCP Protocols are used for Satellite based Networks the effect of wireless channel errors also become significant [16]. Hence this assumption of two state concept does not lead to the most optimal performance. It is obvious from the above mentioned reasons that use of TCP over Satellite channel need to address equally the effect of congestion and channel noise which are important detrimental factors for performance degradation [13][21]. To handle the problem squarely one need to consider Multi State representation of network condition. There is a need to develop a model to handle and represent Multi State network conditions for satellite based TCP/IP networks. In order to formulate the model and understand the various system parameters which control it, the model need to be explicitly evolved and analyzed.

The inferences from this analysis as discussed in this paper are used in an innovative way to work out an Adaptive Multi-State Proactive Transport Protocol with a view to improve the overall network performance over a Satellite channel which has high degree of channel noise and appreciable congestion. In order to formulate the Multi-State Network classification model detail simulation based experiments in ns2 [12] have been carried out to ascertain the combined effect of congestion and channel error on the RTT values pertaining to a connection. The inference from these simulation experiments has been used in the evolution of a Statistical model for network state classification based on RTT values.

The paper is organized as follows. We introduce the RTT Analysis for Multi State Model in Section 2. In Section 3, we propose the statistical formulation of model parameters. Section 4 describes the hierarchical network state classification method. Section 5 describes the Adaptive Multi State Proactive Transport protocol. Section 6 describes the design considerations of the proposed protocol. Section 7 details the Test and Evaluation methodology. Finally, in Section 8 we conclude the paper.

\section{RTT ANALYSIS FOR MULTI STATE MODEL}

The primary goal of the model is to carry out multi state classification of network states with a view to differentiate (i) No Congestion No Error, (ii) No Congestion High Error, (iii) High Congestion No Error and (iv) High Congestion High Error conditions of the network. This will be a significant departure from the conventional two state network classification model with states (i) No Congestion and (ii) Congestion. Using the four states model the combined effect of congestion and corruption on the network can be ascertained. This model will be primarily worked out to handle the performance degradation of TCP over satellite channel where there is coexistence of channel noise and congestion. For the formulation of the model simulation based experiments have been carried out in ns2 [12] to study and analyze the impact of congestion and channel errors on the model parameters. The inference of these analysis will help us in understanding a set of network system parameters which can be properly incorporated in the proposed Adaptive Multi State Proactive Transport Protocol.

\subsection{RTT based Modelling Philosophy}

When a TCP connection is in progress, the sender has no idea about what is happening in the network. It only receives the ACKS, sometimes duplicate ACKS and encounters timeout when the ACKs do not come within an anticipated time[22]. From the received ACKs, the RTT is the 
only information available to the sender which can be used for a proactive decision making regarding the state of the network.

\subsection{Use of RTT Mean}

The RTT in general is considered to be an independent random variable [9]. While a TCP connection proceeds, if we measure the individual RTT values for every window for all the segments transmitted in that window, it will not convey much information regarding the condition of the network, as the transient RTT values are dependent on many dynamic network parameters which changes so frequently that no stable conclusion can be drawn from them. Moreover as suggested in [19], the frequency with which we sample the RTT values is generally less than the Nyquist's Sampling frequency requirement if we consider the RTT values as a time varying signal. So as the sampling is done at a rate lower than needed a proper reconstruction of the signal will not be possible and there is a chance to respond to values corrupted with noise. Another important point is that in a network there are many connections sharing a common bandwidth, so an increase or decrease in RTT cannot be attributed to be happening because of that particular connection and taking action on the individual RTT values may not generate optimal conclusion [19][23]. So we have considered the mean of all the RTT values in a window, which convey more information or may be thought of as a more representative value of the RTT for that specific window to be used in our RTT based model.

\subsection{Simulation based Experimental Results}

In this paper, we have performed some simulation based experiments using ns2 [12] with the simulation setup of 10 senders transmitting to 10 receivers over a GEO link as described in Section 7, to find out the impact of network congestion and packet error rates on the RTT values. These simulation based experiments are needed in the formulation of the Multi State representation of network condition. Three classes of experiments have been performed, (i) considering an uncongested network, (ii) considering a congested network and (iii) progressive levels of network congestion but with very less channel errors

\subsection{Analysis of Results}

In Fig. 1 to Fig. 9 the mean RTT values are plotted with simulation time for a TCP connection as the simulation progresses. First, we have considered the packet error rate to be 0 and it can be seen from Fig. 1 that the mean RTT remains in a closed range of 560 to $570 \mathrm{~ms}$. On the contrary in Fig. 5 even with PER 0, the mean RTT values are more dispersed which shows the effect of congestion on the RTT values. In both the network conditions we have gradually increased the packet error rate from PER 0.001 to PER 0.1 and it has been observed that as we increase the PER for uncongested network the values are seen to have more frequent flickers and dispersion increases. In a congested network condition the effect is more pronounced than its uncongested counterpart for the same PER value. It has also been observed that congested network condition leads to an increase in the DC value of the concerned parameter, larger dispersion and flickering of mean RTT. In Fig. 9 to ascertain the effect of congestion on mean RTT values we have intentionally infused higher levels of congestion in the network, by chocking the available bandwidth to the satellite to lower values of $4 \mathrm{Mbps}, 3 \mathrm{Mbps}$ down to $1 \mathrm{Mbps}$ where the optimum bandwidth required is $5 \mathrm{Mbps}$ as described in Section 7 in detail. Fig.9 directly depicts the high correlation, the DC value of the RTT mean has with increasing levels of congestion. The simulation experiment used a GEO satellite link having RTT around 550 to $570 \mathrm{~ms}$. In the simulation scenario decreasing bottleneck bandwidth between the two earth stations the individual maximum bandwidth shared by the connections can be though of as reducing from $500 \mathrm{kbps}$ to $100 \mathrm{kbps}$. The mean RTT values are seen to increase and to be hovering in the range of $570 \mathrm{~ms}, 700 \mathrm{~ms}, 900 \mathrm{~ms}, 1400 \mathrm{~ms}$ and $2700 \mathrm{~ms}$ corresponding to the decrease in link capacity and increasing degree of congestion [22][24]. The inferences which can be drawn from 
International Journal of Computer Networks \& Communications (IJCNC) Vol.2, No.6, November 2010

these experiments are given below as (i) From the first experiments it can be concluded that no congestion and no error leads to very less dispersion of the mean RTT values, dispersion increases with increasing PER (ii) From second experiment it can be concluded that under

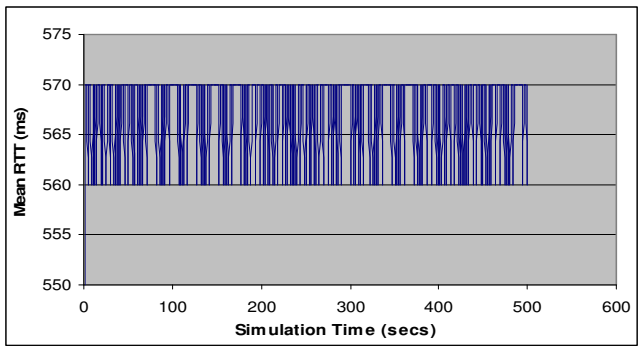

Fig. 1 PER $=0.00$ Queue $=20$ Max Receiver Win $=20$

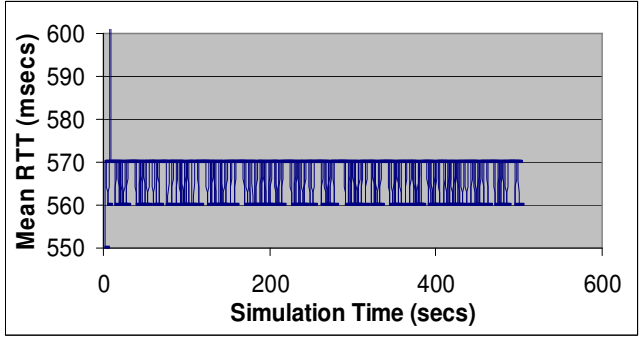

Fig. 2 PER $=0.001$ Queue=20 Max Receiver Win= 20

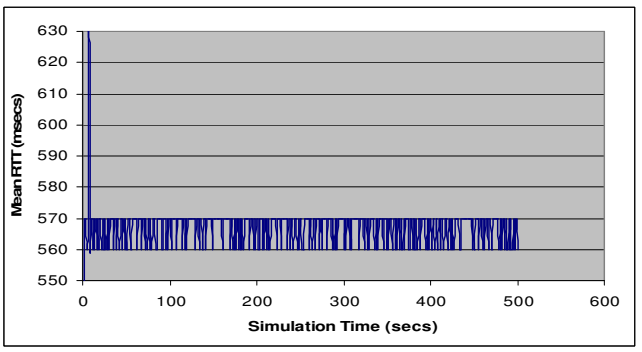

Fig. 3 PER $=0.01$ Queue $=20$ Max Receiver Win $=20$

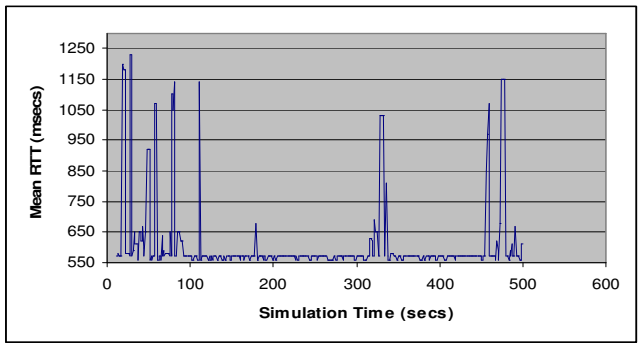

Fig. 4 PER $=0.1$ Queue $=20$ Max Receiver Win $=20$

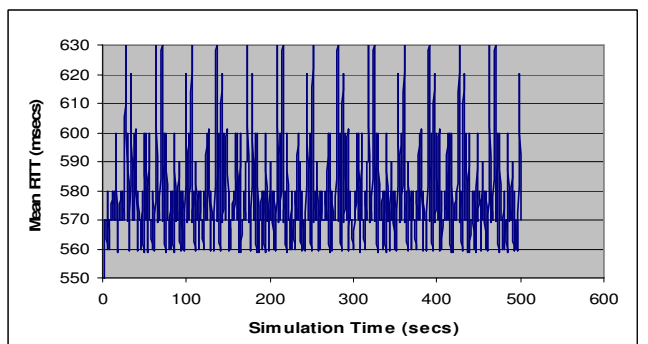

Fig. 5 PER $=0.00$ Queue $=64$ Max Receiver Win $=64$

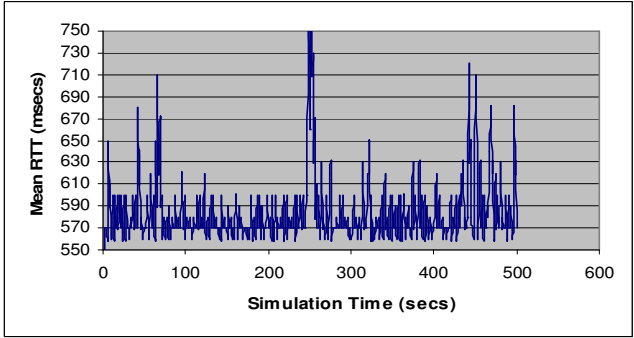

Fig. 6 PER $=0.001$ Queue $=64$ Max Receiver Win $=64$

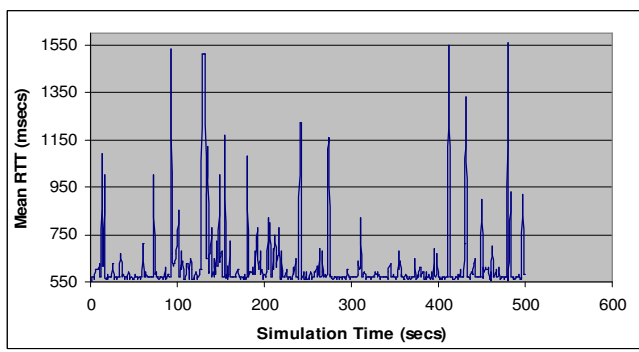

Fig. 7 PER $=0.01$ Queue $=64$ Max Receiver Win $=64$

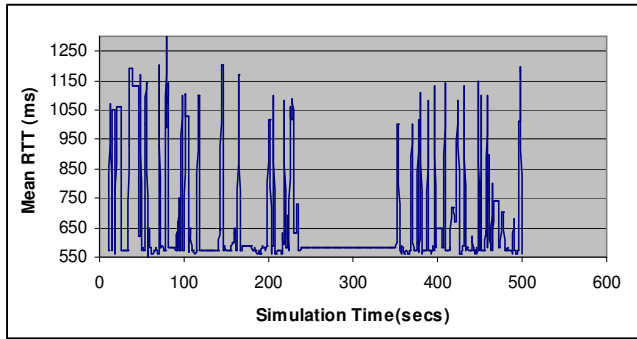

Fig. 8 PER $=0.1$ Queue $=64$ Max Receiver Win $=64$ 


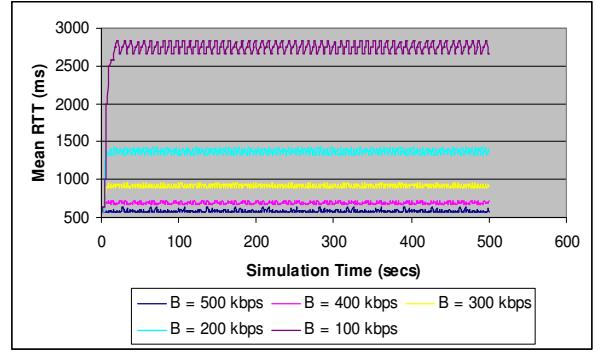

Fig. 9 RTT Mean Variation For Different Congestion Levels

congested condition, the increase in packet error rate leads to more dispersion of mean RTT values. (iii) From the third experiment it can be concluded that if channel error is very less the degree of congestion is directly related to the increase in DC value of the RTT Mean. So it can be concluded that RTT has multivariate correlation [23] with congestion and PER prevailing in the network.

\section{STATISTICAL FORMULATION OF MODEL PARAMETERS}

In this section, considering this inference discussed in Section2 we have developed a model to classify the short term and long term characteristics of a network by estimating the presence and absence of congestion and corruption in the network. Considering the combinatorial combination of the presence of congestion and corruption as binary variable 0 and 1 , the network can be considered to be in one of the four state $00,01,10,11$ where, state 00 signify No Congestion No Error, state 01 signify No Congestion High Error, state 10 signify - High Congestion No Error and state 11 signify - High Congestion High Error

Depending on the dynamics of the data passing through the network [24], error encountered during the transfer process and the reaction of the congestion control process towards the undelivered packets the network moves through the above mentioned states. From the available RTT values we have intended to determine certain statistical parameters, which represent the extent of congestion \& corruption in the network [22]. These parameters when combined through a hierarchical decision making process helps in classifying the network into the above mentioned states. The classification model using the statistical parameters along with their definitions is presented below.

\subsection{RTT based Network State Classification Model}

The total duration of a TCP connection can be visualized to be divided into a series of windows, [9] where at the start of each window the number of TCP segments to be transmitted in that window is governed by the value of the prevailing congestion window (cwnd). TCP sends all the segments up to cwnd and waits for acknowledgment of the segments to return to determine the value cwnd for the next window. So for a given window, there will be a series of RTT values obtained corresponding to all packets transmitted in that window.

Lets us assume that there are $\mathrm{m}$ number of windows in the duration of a TCP connection denoted by $\mathrm{W}_{1}, \mathrm{~W}_{2} \ldots \mathrm{W}_{\mathrm{i}} \ldots \mathrm{Wm}$. Let us define the following variables

$\mathrm{W}_{\mathrm{i}}$ denote the ith window, $0<\mathrm{i}<\mathrm{m}$, cwnd $_{\mathrm{i}}$ denote the value of cwnd for the ith window, $R T T_{i, j}$ is the RTT measurement for the jth packet for the ith window, $0<\mathrm{i}<\mathrm{m}, 0<\mathrm{j}<$ cwnd $_{\mathrm{i}}$

So we have a series of RTTi,j values corresponding to each window Wi, which are the independent values from where we try to define the following variables 


$$
M W_{i}=\frac{\sum_{j=0}^{c w n d}{ }^{i} R T T_{i, j}}{c w n d_{i}}
$$

MWi denote the mean of the RTT values for the ith window Wi

$$
M_{k}=\frac{\sum_{j=i-k}^{i} M W_{j}}{k}
$$

$\mathrm{M}_{\mathrm{k}}$ denotes the window average of the mean values $\mathrm{MW}_{\mathrm{j}}$ for the last $\mathrm{k}$ windows.

$$
M R_{k}=\frac{M_{k}-I R T T}{I R T T}
$$

$\mathrm{MR}_{\mathrm{k}}$ denote the normalized rise of the mean RTT values from the ideal RTT values. IRTT can be determined from previous knowledge of the end to end delay and is given by the minimum of all RTT values measured for that specific connection. So IRTT in the beginning will be equal to the RTT values obtained through the SYNC message exchanged during connection setup and gradually can be refined with new values. $\mathrm{MR}_{\mathrm{k}}$ is an estimation of the short term condition of the network load. MWi denote the mean of the RTT values for the ith window Wi.

$$
M_{i}=\frac{\sum_{j=0}^{i} M W_{j}}{i}
$$

In (2) if we replace $k$ with $i$ then we get the long term mean $M_{i}$ which is equal to the mean of all the mean values $\mathrm{MW}_{\mathrm{j}}$ obtained for each window from the beginning. So the $\mathrm{M}_{\mathrm{k}}$ values give the short term characteristics of the network and $\mathrm{M}_{\mathrm{i}}$ gives the long term characteristics as it is the integration of all the values the connection has experienced from its inception. Similarly $\mathrm{MR}_{\mathrm{i}}$ denotes the long term mean rise as given below where i denote the prevailing window. So as the connection progresses, at each instant if the prevailing window is $i$, then $M_{i}$ denotes the rise of the mean from the ideal value considering values from beginning of connection.

$$
M R_{i}=\frac{M i-I R T T}{I R T T}
$$

Next we calculate the standard deviation of the mean rtt values, $\mathrm{MW}_{\mathrm{j}}$ for the last $\mathrm{k}$ windows,

$$
\sigma_{k}=\sqrt{\frac{\sum_{j=i-k}^{i}\left(M W_{j}-M_{k}\right)^{2}}{k}}
$$

Here $\mathrm{k}$ denotes the number of last window values considered.

$$
\begin{aligned}
& \sigma_{i}=\sqrt{\frac{\sum_{j=0}^{i}\left(M W_{j}-M_{i}\right)^{2}}{i}} \\
& R_{k}=\frac{\sigma_{k}}{I R T T}
\end{aligned}
$$

$\mathrm{R}_{\mathrm{k}}$ denotes the ratio of the standard deviation to the ideal RTT which signifies the extent to which the RTT values have been dispersed from the ideal condition. $\mathrm{Ri}$ is the long term ratio.

$$
R_{i}=\frac{\sigma_{i}}{\operatorname{IRTT}}
$$

The parameter $S_{k}$ denotes the ratio in which the intermediate Mean $R T T$ values $M_{j}$ and $M_{j+1}$ 
have not changed beyond a threshold $\alpha$ to the total number of windows considered. In our case $\alpha$ is taken as $1 \%$ of previous RTT Mean so, $\alpha=0.01 * \mathrm{MWj}$. $\mathrm{S}_{\mathrm{k}}$ denotes the short term stability of the network.

When $\mathrm{k}$ is replaced by $\mathrm{i}, \mathrm{S}_{\mathrm{i}}$ denotes the stability of the connection from the beginning. In the RTT based Model for network state classification we have defined some statistical parameters from the measured $\mathrm{rtt}$ values which convey different meaning about the short term and long term characteristics of the network. So corresponding to each window Wi we can think of the set of values $\left\{\mathrm{MR}_{10}, \mathrm{MR}_{20}, \mathrm{MR}_{30}, \mathrm{MR}_{\mathrm{i}}\right\}$ for Mean Rise, the set $\left\{\mathrm{R}_{10}, \mathrm{R}_{20}, \mathrm{R}_{30}, \mathrm{R}_{\mathrm{i}}\right\}$ for Ratio and for stability $\left\{S_{10}, S_{20}, S_{30}, S_{i}\right\}$ to signify the short term and long term characteristics of the network. The way these parameters varies under different network state is shown below.

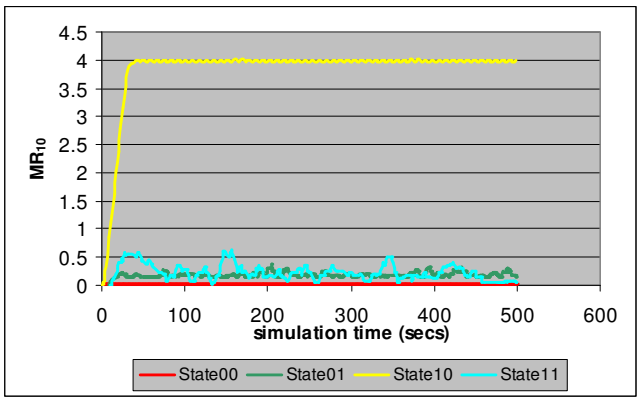

Fig. 10 Mean Rise Window Average Length $=10$

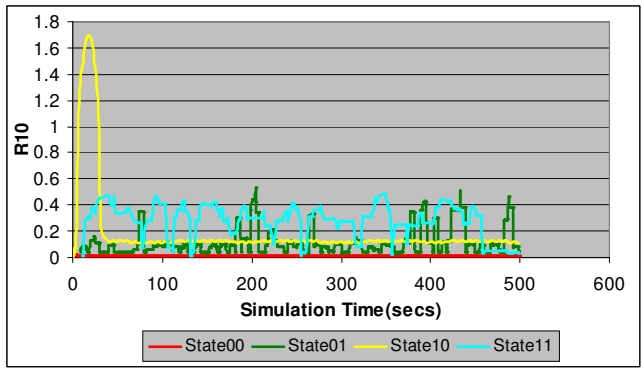

Fig. 11 Ratio Window Average Length $=10$

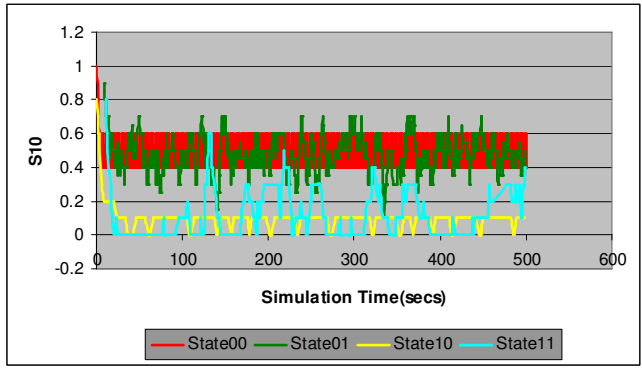

Fig. 12 Stability Window Average Length $=10$

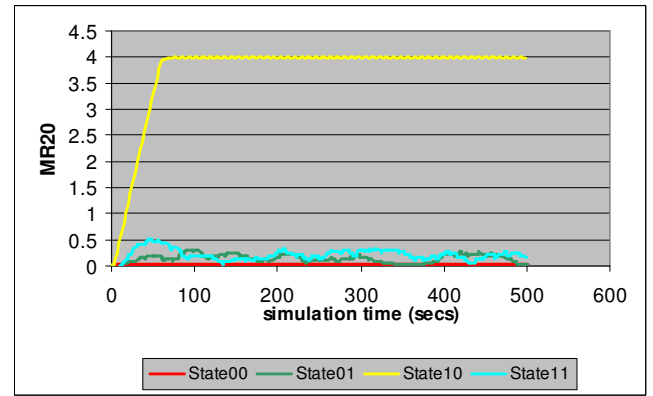

Fig. 13 Mean Rise Window Average Length $=20$

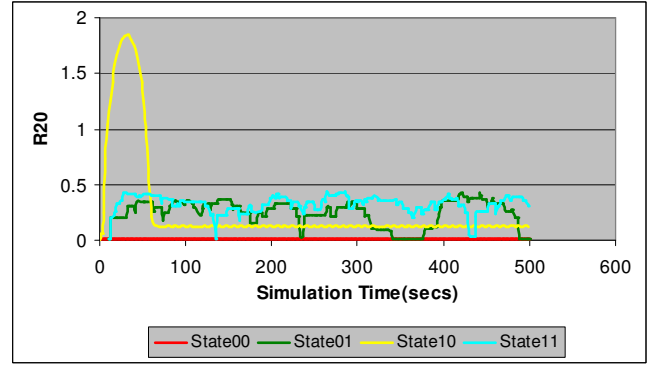

Fig. 14 Ratio Window Average Length $=20$

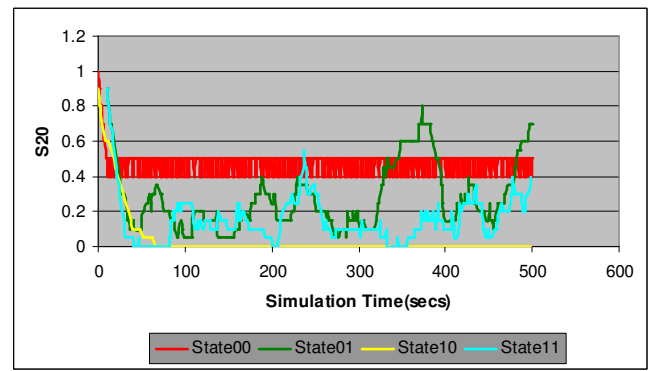

Fig. 15 Stability Window Average Length $=20$

\subsection{Short Term Network Characteristics}

The short term characteristics of the network can be obtained by considering a window average of some fixed number of past windows. This will give us a more recent condition of the network. We have simulated considering the last 10,20 and 30 windows which are displayed in the graph below. All the three parameters considered in the protocol like mean rise, ratio and stability is seen to vary as shown in Fig. 10 to Fig. 18. 


\subsection{Long Term Network Characteristics}

From the Fig. 10 to Fig. 21 it can be seen that as the value of the parameter k increase from 10 to 20 to 30, the standard deviation of all the individual plotted statistical parameters is seen to reduce. This is obvious considering the principle of the sampling theory, that a larger sample reduces the standard deviation of the sample mean and leads to a more accurate estimation of the population concerned. So, the long term estimate $\mathrm{MRi}, \mathrm{Ri}$ and $\mathrm{Si}$ is seen to have much less dispersion in Fig. 19, Fig. 20 and Fig. 21 as the simulation time progresses, thereby characterizing the network into its different states. So the variable MRi, Ri and Si can be considered as the network state variables and for each progressive window, get updated as per the equations described above.

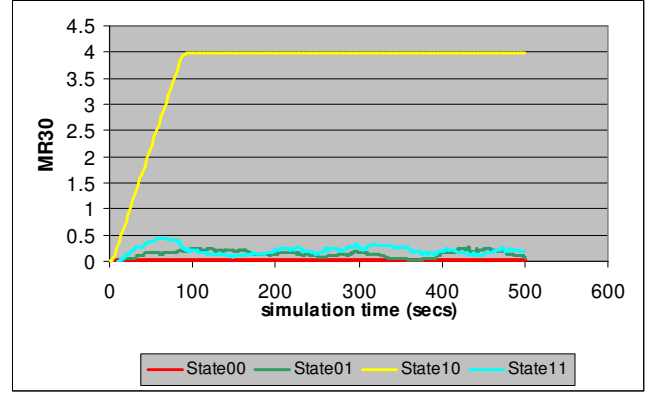

Fig. 16 Mean Rise Window Average Length $=30$

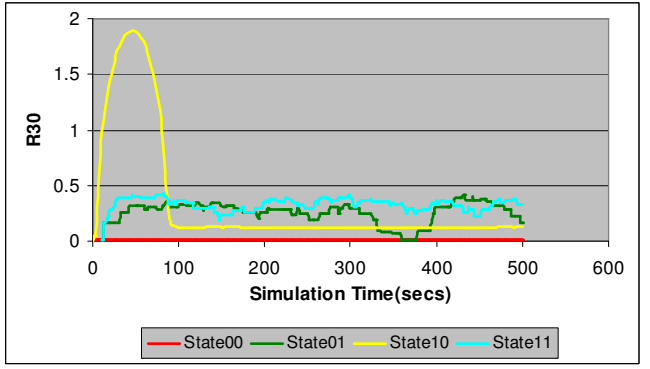

Fig. 17 Ratio Window Average Length $=30$

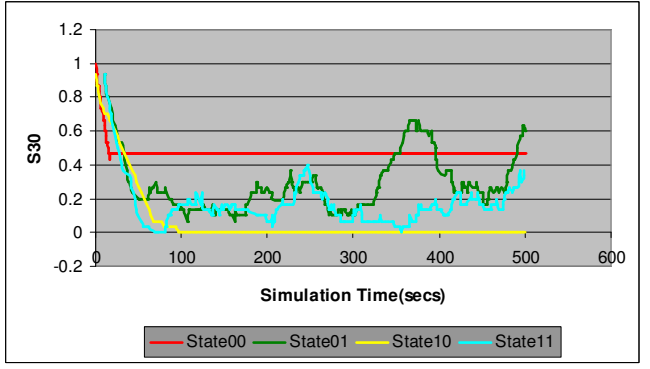

Fig. 18 Stability Window Average Length $=30$

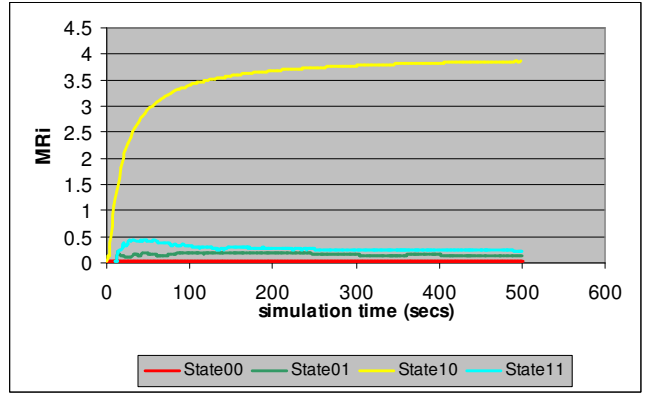

Fig. 19 Mean Rise Window Average Length $=\mathrm{i}$

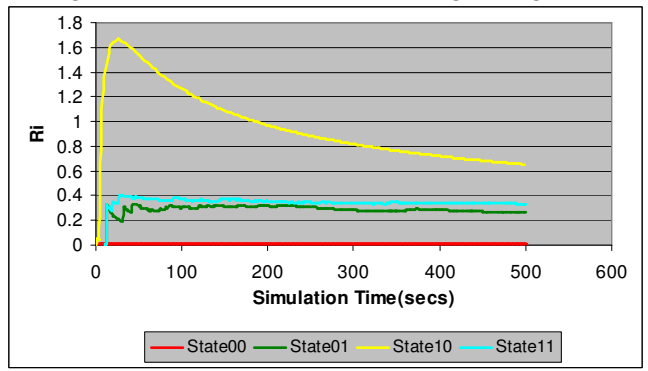

Fig. 20 Ratio Window Average Length = i

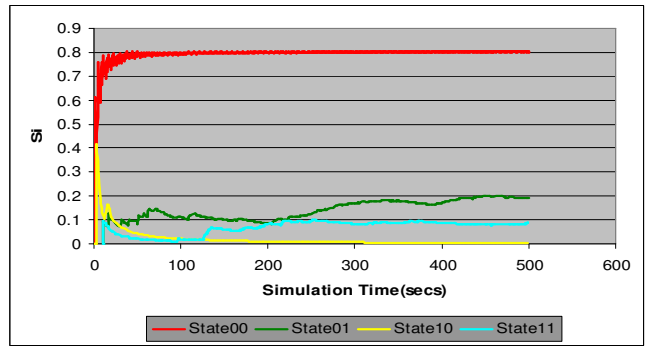

Fig. 21 Stability Window Average Length $=\mathrm{i}$

\section{HIERARCHIAL NETWORK STATE CLASSIFICATION}

This section describes the network state classification logic along with the classification of the statistical parameters described in Section3. The equations to derive the thresholds have been explained considering values derived from simulation experiments. Table I, Table II and Table III contain values of parameter $\mathrm{MRi}, \mathrm{Ri}$ and $\mathrm{Si}$ respectively calculated from simulations, considering the scenario described in Section 7 for a Geo Satellite Network. In Section 5 it will be shown how the Parameter Adaptation Algorithm automatically generates the Tables described in this section. A pictorial representation of the parameter classification logic along with multi state hierarchical network classification logic is also presented in this section. 


\subsection{Mean Rise Classification}

Table I gives the minimum and maximum value obtained by the Mean Rise, MRi parameter through the simulation experiment under different network states.

TABLE1

\begin{tabular}{ccccc}
\multicolumn{5}{c}{ MEAN RISE VALUES FOR GEO NETWORK (MRi) } \\
\hline State & 00 & 01 & 10 & 11 \\
\hline Min & 0.031 & 0.1 & 0.40 & 0.20 \\
\hline Max & 0.10 & 0.2 & 3.96 & 0.50 \\
\hline
\end{tabular}

Let us define the following thresholds LMRi and HMRi which differentiate the MRi value into low $(\mathrm{L})$, medium $(\mathrm{M})$ and high $(\mathrm{H})$ ranges as shown in Fig. 22

$$
L M R_{i}=\operatorname{Max}\left\{M R_{i} \text { State } 00, M R_{i} \text { State } 01\right\} \quad(10) \quad H M R_{i}=\operatorname{Max}\left\{M R_{i} \text { State } 1\right\}
$$

LMRi denotes the lower threshold is given by the max value obtained by MRi in 00 and 01 states. So when the value of MRi is below this threshold we can assume that the network is in 00 or 01 state. HMRi denotes the upper threshold is given by the max value obtained by MRi in state 11. When the value of MRi is between LMRi and HMRi the network is estimated to be either in state 10 or 11. MRi values higher than HMRi denotes the high congestion no error state of 10. Fig. 22 shows the classification of network states using MRi.

\subsection{Ratio based Classification}

The following table gives the minimum and maximum value obtained by the Ratio parameter Ri through the simulation experiment. We define a threshold

$$
L R_{i}=\operatorname{Max}\left\{R_{i} \text { State } 00\right\}
$$

TABLE II

\begin{tabular}{ccccc}
\multicolumn{5}{c}{ RATIO VALUES FOR GEO NETWORK (Ri) } \\
\hline & 00 & 01 & 10 & 11 \\
\hline Min & 0.013 & 0.23 & 0.179 & 0.35 \\
\hline Max & 0.2 & 0.3 & 1.2 & 0.40 \\
\hline
\end{tabular}

LRi is used as a threshold which divides the ratio values into two broad cases low (L) and high $(\mathrm{H})$ and any value lower than the threshold can be considered to be a sign that the network is in state 00 . If values higher than this are obtained then the network can be in either of the states 01 , 10 and 11 as shown below.

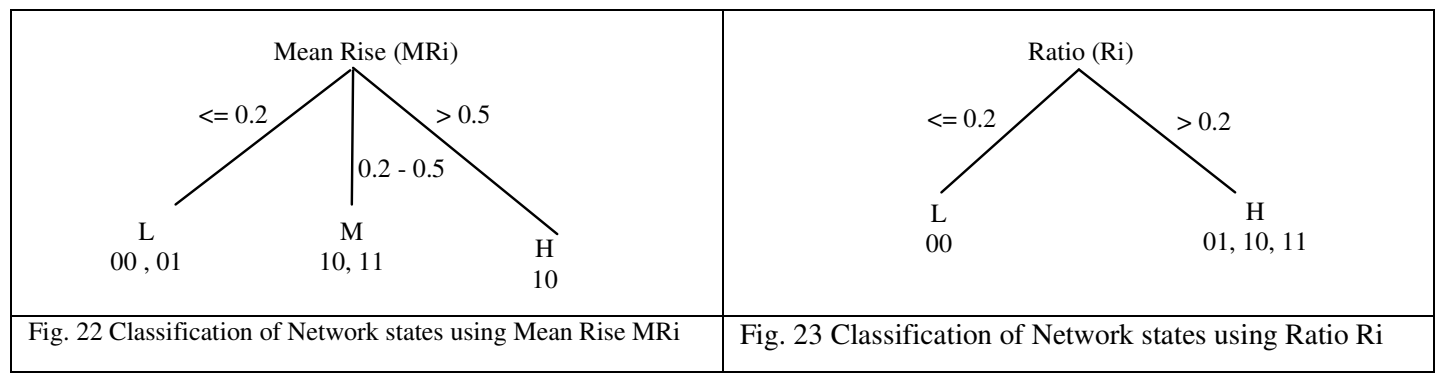

\subsection{Stability based Classification}

The following table shows the values of the stability parameter obtained through simulation experiment. We can define some threshold, which differentiate the stability parameter into Low (L), Medium (M) and High $(\mathrm{H})$ values classifying the network into states as shown in the Fig. 24. Let us define the thresholds

$L S_{i}=\operatorname{Max}\left\{S_{i}\right.$ State 10$\}$

$$
H S_{i}=\operatorname{Min}\left\{S_{i} \text { State } 00, S_{i} \text { State } 01\right\}
$$


TABLE III

STABILITY VALUES FOR GEO NETWORK ( $\mathrm{Si})$

\begin{tabular}{ccccc}
\hline & 00 & 01 & 10 & 11 \\
\hline Min & 0.40 & 0.16 & 0.00 & 0.02 \\
\hline Max & 1.0 & 0.4 & 0.02 & 0.16 \\
\hline
\end{tabular}

Si values lower than LSi will be considered low denoted by $\mathrm{L}$ which is typically seen in state 10 and value between LSi to HSi is typically seen in state 11 and considered to be medium denoted by $\mathrm{M}$ and values higher than HSi means a high stability factor denoted by $\mathrm{H}$ typically seen in state 00 and 01 .

\subsection{Classification combining all Parameters}

For the classification process we need to determine the threshold values for all the parameters described MRi, Ri and Si. For a type of network like GEO satellite network the values of the parameters can be predetermined [20]. For a more accurate threshold calculation there has to be an adaptive way of finding out the thresholds values. The protocol has to be run on the network concerned for a sufficiently long span of time in predetermined network conditions. The values of the different parameters for all these different network conditions can be stored till the parameters become stable or their standard deviation is almost zero. It has been seen in Fig. 19, Fig. 20 and Fig. 21 how the parameters lead to stable value conveying long term characteristics of the network. Once these stable values of these parameters are obtained the classification thresholds can be determined using the equations (10) to (14) discussed above. Now we define the classification approach where in the beginning the network is considered to be in any one of the four states $00,01,10,11$, Then we check the MRi value and the estimated state is partitioned to $\{00,01\},\{10,11\}$ or 10 state depending on whether the mean rise is very less, moderate or high. A very high mean rise is only seen in state 10 so in case a very high value is obtained it can be directly inferred that the state is 10 . For other cases to be more certain a checking is done with the Ri parameter. A low and high value of Ri partitions the set $\{00,01\}$ to 00 and 01 respectively and set $\{10,11\}$ becomes unpredictable if $\mathrm{Ri}$ is low. To be more precise we check for the stability parameter Si to be high with state 00 and 01 . For the set $\{10,11\}$ the Si parameter classifies it to state 10 when low and state 11 when having moderate values. In cases the proper combination of the parameters are not found the network is said to be unclassified which is shown by $\mathrm{X}$ in Fig. 25. In this context one point is worth mentioning that if more number of unclassified states are arrived from the network classification algorithm that signifies that the threshold value calculated are not properly tuned so a recalculation is needed in that case.

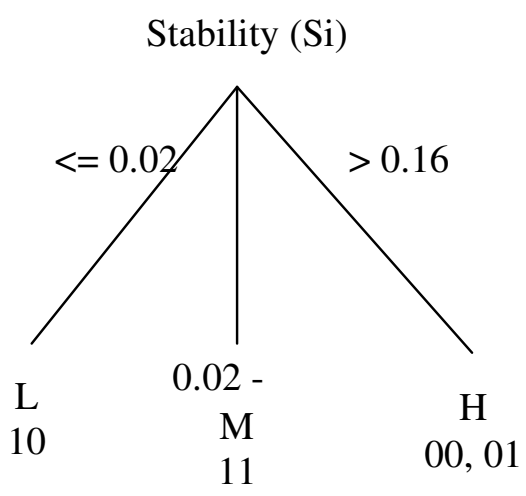

Fig. 24 Classification using Stability Si

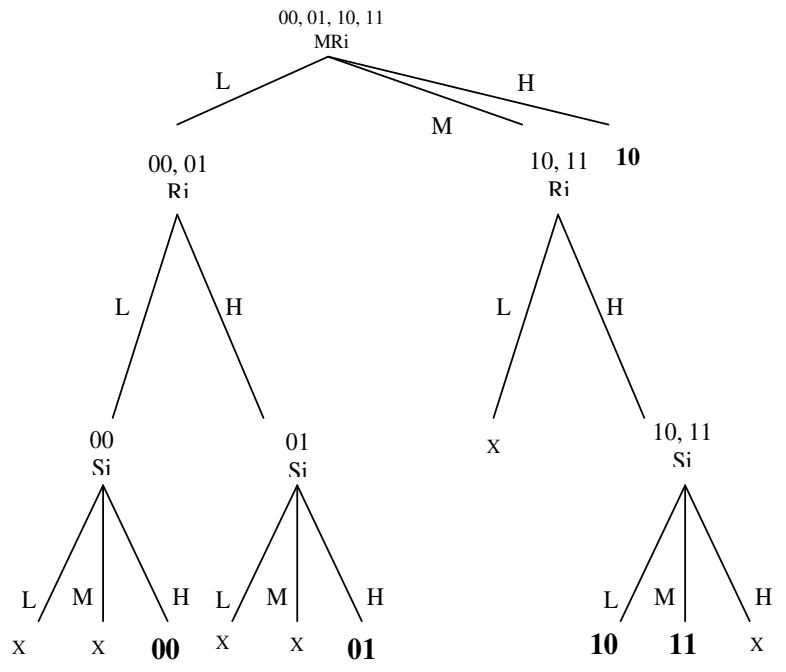

Fig25. Classification combining $\mathrm{MRi}, \mathrm{Ri}$ and $\mathrm{Si}$ 


\section{ADAPTIVE MULTI STATE PROACTIVE TCP PROTOCOL}

\subsection{Brief Working of Adaptive Multi-State Proactive TCP}

In this section we introduce an Adaptive Multi State Proactive Transport Protocol, which is an end-to-end solution to improve the throughput performance in satellite networks [15][21]. The protocol uses a proactive approach and is composed of new approaches like Proactive Slow Start, Proactive Congestion Avoidance, State based Error Recovery, and State based Timeout Action algorithms along with traditional TCP algorithms like Fast Retransmit [20]. The philosophy of the protocol is that certain statistical parameters of the network can be slowly adapted for better proactive actions. With a prior knowledge about the characteristic of the network the performance of the protocol can be greatly enhanced specially during challenging environments [13][15][21] of high wireless link errors. In Fig. 26 the overall mechanism of the proposed Adaptive Multi-State Proactive TCP scheme is depicted.

The Network State Classification Algorithm is the heart of this Adaptive State based protocol. For every window after the ACKs for all the transmitted packets are received before the start of a new round of packet transfer, Network State Classification is performed which estimates the state in which the network is presently or an unclassified state using the Network Classifiaction Algorithm as described in Fig 31.

The protocol starts with the Proactive Slow Start phase where the slow start threshold is kept at half the receiver window, the congestion window to one and the state of the protocol is, No Congestion No Error ie state 00. The receiver window signifies the maximum number of segments the receiver can accommodate. The congestion window is increased by one with each received ACK so that the congestion window doubles every RTT until the slow start threshold is reached. But the mean RTT is also checked during this phase and if RTT increase for three successive windows [20] is detected the congestion window is reduced by an Adaptive penalty factor shown in Fig. 40. After slow start threshold is crossed the protocol moves to the Proactive Congestion Avoidance phase where it increases the congestion window by $1 / \mathrm{cwnd}$ for every reception of $\mathrm{ACK}$ as in traditional Congestion Avoidance Algorithm. Now if an acknowledgment is not received for a transmitted segment within its retransmission timeout period, the timer expires and the algorithm enters the State based Timeout Action phase. As shown in Fig. 37 here depending on the state of the network the timeout action is taken. If the state is any among 00, 10 or 11 then the congestion window is reduced to one and Proactive Slow Start is initiated [18]. If the state is 01 then the congestion window is not reduced and the protocol remains in Proactive Congestion Avoidance, only the packets not acknowledged are retransmitted.

During the Proactive Congestion Avoidance the mean RTT is checked every time and if an increase in RTT for three successive congestion windows [20] is detected the congestion window is reduced by the adaptive penalty factor using State based Proactive Action Algorithm as shown in Fig. 41. For every window the Network State Classification also predicts the state of the network.

Now if three duplicate ACKs are received the protocol calls the Fast Retransmit algorithm and a retransmission [18] is done of the lost segment and the protocol moves to the State based Error Recovery Algorithm as shown in Fig. 39. In this phase if the protocol is in state 10 or 11 which signals that the network has some congestion and a packet is not received by the receiver then the chance of the packet being lost by congestion is more. So the congestion window is reduced to half and protocol moves to the Proactive Congestion avoidance phase. If the state of the network is 00 then the congestion window is reduced to $3 / 4$ of this value. No reduction in congestion window is done when the state of the network is 01 which signifies that the loss is error initiated. An unclassified state is considered as state 11 because in state 11 all actions are conservative and this prevents from performance degradation during unsuccessful classification. The Adaptive Multi State Proactive TCP Protocol uses some concepts of Proactive TCP [20] Protocol designed by the authors and is best suited to work in high error conditions. 


\subsection{Overall Operation of the Protocol}

The overall operation of the protocol can be classified into a Training Phase and an Operational Phase. During the Training Phase the protocol learns about the characteristics of the network by adapting the statistical parameters and calculates the threshold parameters, which are eventually used for network state classification. The basic algorithm is presented below in Fig. 27.

Before the Adaptive Multi State Algorithm can be used it is necessary to have the threshold values for the network state classification process. For a particular type of network these values can be predetermined where the traffic dynamics are less. For example we have calculated the value of the thresholds for a GEO based network in our simulation based experiments with an idea about the ideal RTT value for a GEO satellite which is $550 \mathrm{~ms}[1][2]$. With predetermined threshold values pertaining to the type of network in which the protocol is supposed to run, the training process may be skipped. But for networks, which have a more dynamic pattern of traffic, before the protocol can be used a training of the protocol is necessary to calculate the threshold values of the parameters. This is a familiarization of the network dynamics to the protocol under different conditions of congestion and error. The network has to be run through varying levels of congestion and corruption so as to create the different network states while the parameters are recorded [23]. In an overview the main algorithms to be used are given below in Fig. 27. Basically using the Parameter Adaptation process the data collection will happen followed by Threshold_Calculation Algorithm to calculate thresholds. These two algorithms are part of the initial training process

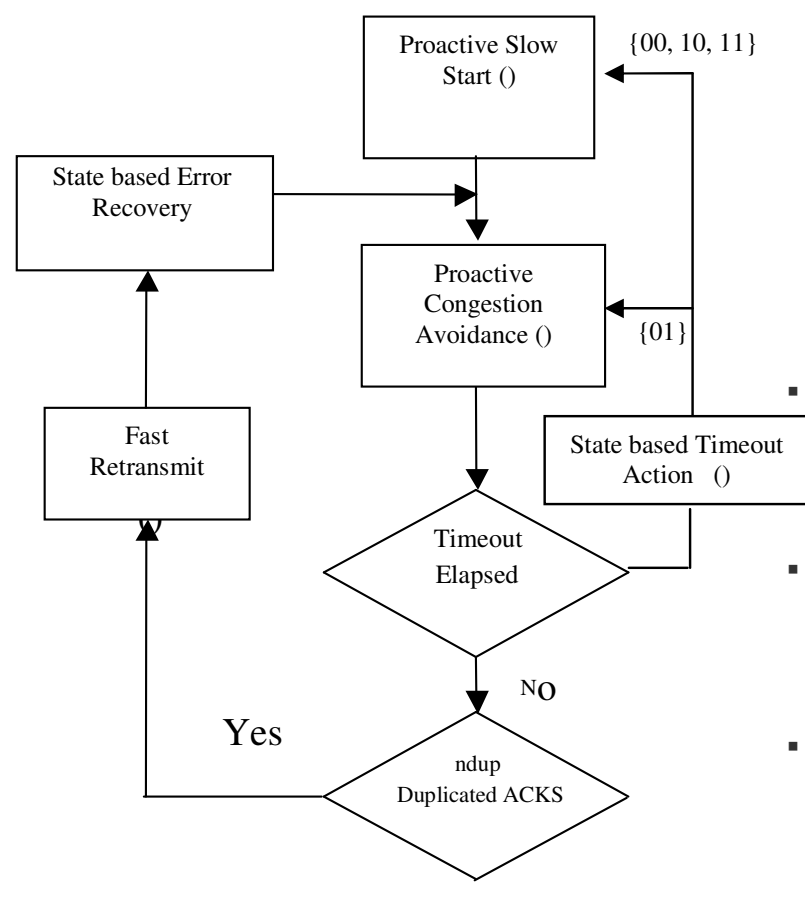

Fig. 26 Flow Start of Adaptive Multi State Proactive TCP
- Once the thresholds are obtained, during the protocol operation the Parameter_Classification Algorithm will be used to classify the status of the parameters. After the classification of individual parameters the Network State Classification Algorithm will predict the network state. This state prediction will be used in different operations of the protocol as discussed later. Training the Protocol has to be done by intentionally varying the levels of congestion and channel errors so as to artificially create network conditions similar to state00, state01, state 10 and state11

The parameters MRi, Ri and Si should be calculated and stored till the values become stable or the standard deviation of the calculated parameters are very less using Parameter_Adaptation()

- Determination of Threshold values like $\{L M R i$, $H M R i\}$ for MRi parameter, \{LRi\} for parameter $R i$, and $\{\mathrm{LSi}, \mathrm{HSi}$ for $\mathrm{Si}$ using Algorithm Threshold_Calculation()

- For each Window after all the transmitted packets are ACKed, the MRi, Ri and Si parameters are updated and classify the Status of Parameters MRi, Si and Ri as Low, Medium or High using Parameter_Classification() Algorithm

- Classify the Network States through a hierarchal checking of the values MRi, Ri and Si according to Fig. 25 and using Network_State_Classification() Algorithm

Fig. 27 Overall Operation of the Protocol 


\subsection{Training Phase of the Protocol}

The input to the Parameter_Adaptation algorithm as shown are the RTT values and the State to which the RTT values corresponds to and the output from this Algorithm are the updated internal Tables of the Protocol which contains the Minimum and Maximum value obtained by each of the parameter MRi, Ri and Si for all the states 00, 01, 10 and 11. The Algorithm in this case calculates the values of the parameters $\mathrm{MRi}, \mathrm{Ri}$ and $\mathrm{Si}$ after each window and stores it until the values of the parameters become stable or their standard deviation is very small. After that only the minimum and maximum values are calculated. It has been seen from Fig. 10 to Fig. 21 how the values become eventually stable. This is needed not to get locked to very high fluctuating values [19] and the long term characteristics of the network can be ascertained.

The input to the Threshold_Calculation Algorithm is the Tables from where the Algorithm outputs the threshold values using equations already discussed. The threshold values are stored internally and updated when the protocol is again run in the training mode to refine the threshold values as necessary.

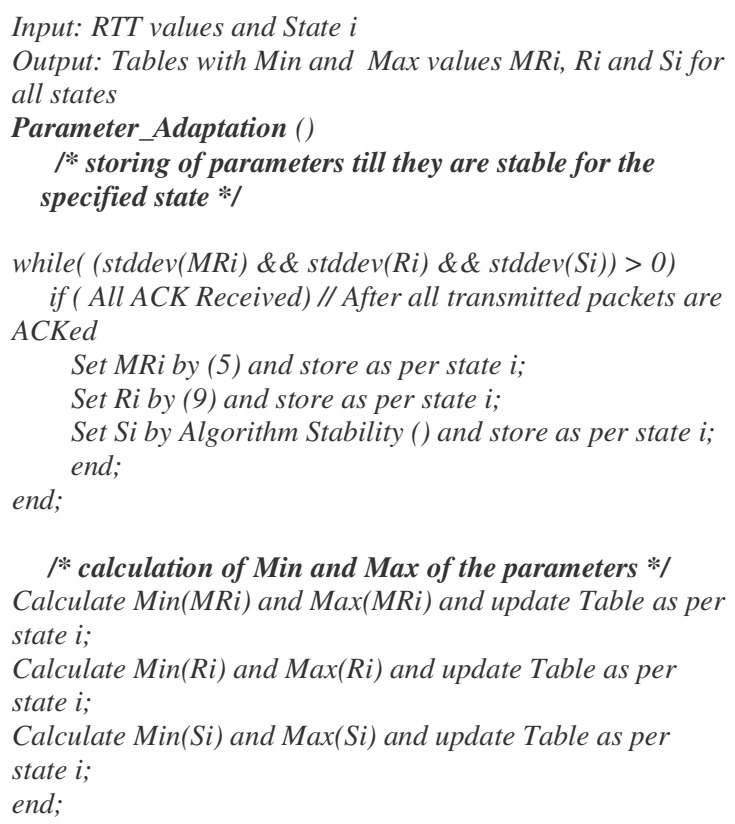

Fig. 28 Parameter Adaptation Algorithm

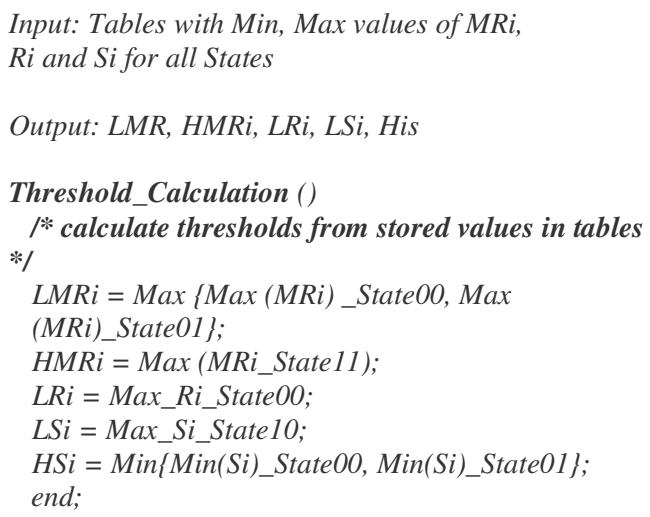

Fig. 29 Threshold Calculation Algorithm

\subsection{Operational Phase of the Protocol}

The Parameter Classification algorithm shown in Fig. 30 classifies the status of the parameters MRi. Ri and Si into low denoted by L, medium denoted by M and high denoted by H. Actually each classification of parameters carries with it an estimation of the possible network state. For example a low value of MRi is possible in state 00 and 01 whereas a medium value is possible in state 10 and 11. A very high value is certainly seen in state10. This is because in state 00 and 01 the load on the network is not that high as compared to state 10 , where only congestion and no error persists. The state 10 appears in medium case also because, when the degree of congestion is less or when during state 10 errors starts happening and the data transmission may be slowed down, the Mean Rise MRi may come in the medium zone. Similarly a low value of Ri can be only possible in state 00 and a high value can be possible in all the other states like 01,10 and 11. This is because both congestion and error in channel increase the standard deviation of the Mean RTT values. Again a very high stability can be only seen in state 00 and 01 where congestion is not there. The lowest stability is seen in the state 10 where the data transmission is maximum and stability the least. A medium value of stability can be seen in state 11 when both congestion and errors persists. So it can be seen that considering a single 
parameter, the network can be estimated to be within a set of the network states. When all these individual estimations are combined the network state classification can be done using Network Classification() algorithm shown in Fig. 31.

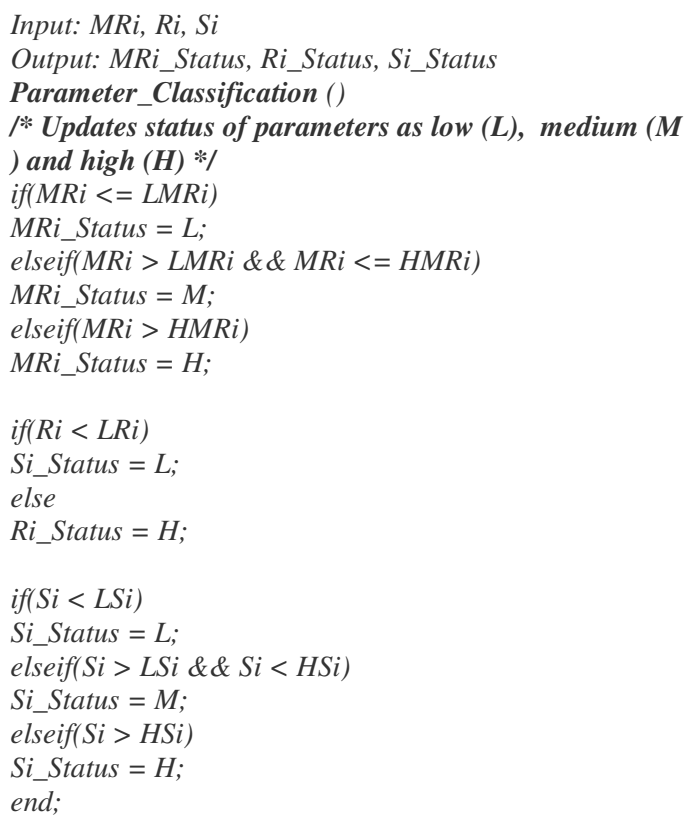

Fig. 30 Parameter Classification Algorithm

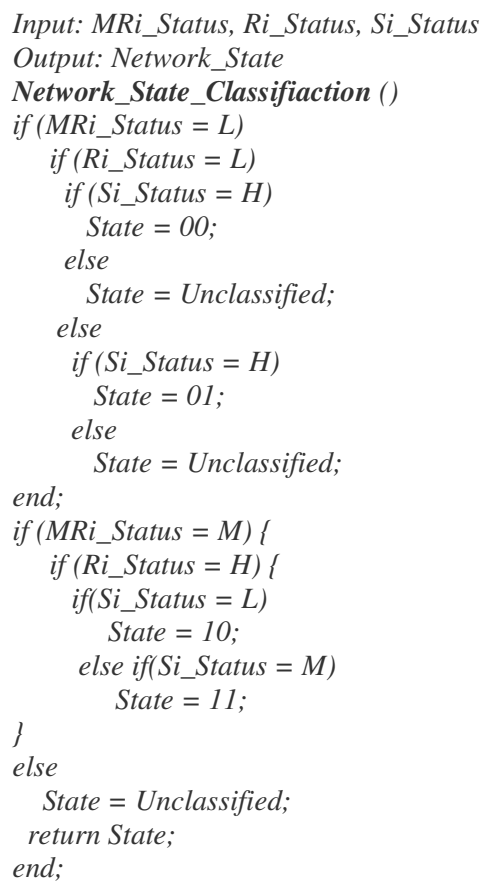

Fig. 31 Network State Classification Algorithm

\section{DESIGN CONSIDERATIONS OF ADAPTIVE MULTI STATE PROACTIVE TCP}

This section describes the internal detail of the proposed protocol and also discusses the rationale of the specific approach. The section describes the Simulation based experiments which have been carried out to analyze in detail how the different protocol related parameters respond to packet error rates with a view to enhance the performance [13][14] of the protocol under high error conditions.

\subsection{Selective Acknowledgment Scheme}

Whenever there is a loss due to duplicate acknowledgment or due to timeout [18], generally transport protocols transmit all the packets again starting from the point the highest acknowledgment was received if cumulative ACK scheme is used. When multiple packet losses appear in the same window the repeated transmission of packets already transmitted leads to some performance degradation [13][16]. So in the Adaptive Multi State Proactive TCP Selective ACK [6] scheme is used, where selectively packets can be retransmitted.

\subsection{Effect of Packet Error on Protocol Parameters}

In the simulation experiment using scenario of Section7, the Packet Error rates is gradually increased from a very low value of 0.0001 to very high value of 0.1 and the total number of Windows, RTT Increase Frequency, DUP ACK Frequency, Timeout Frequency and achieved throughput is plotted as shown in Table IV. The maximum bandwidth utilization possible for each connection is 500kbps if they are fairly [11] [10] sharing the 5Mbps of Satellite bandwidth available. 
Fig. 32 shows the total number of windows or in other words rounds [9] which could be completed within the 500 secs simulation time. It can be seen that as the packet error rate increases, due to more retransmissions the protocol is able to complete lesser number of rounds of data transfer. This parameter is more useful to visualize the amount of time the protocol keeps waiting because of an increase in the retransmission timer. In PER of 0.0001 within a 500 secs time, 842 rounds could be completed and in PER of 0.1 only 318 rounds could be completed. This shows why the throughput is degraded as the PER increases.

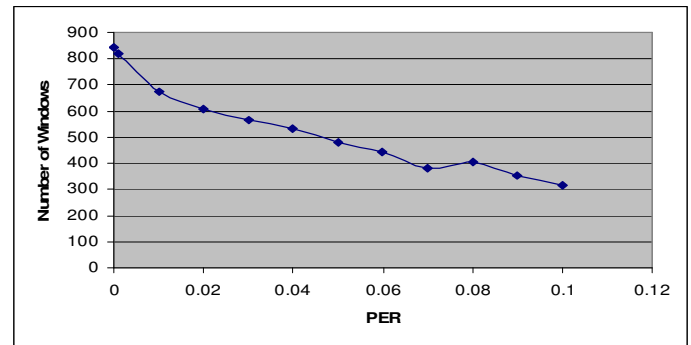

Fig. 32 Number of Windows vs PER

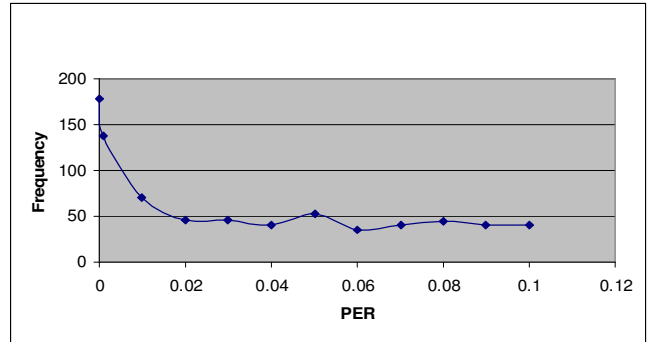

Fig. 33 RTT_Increase_Frequency with PER

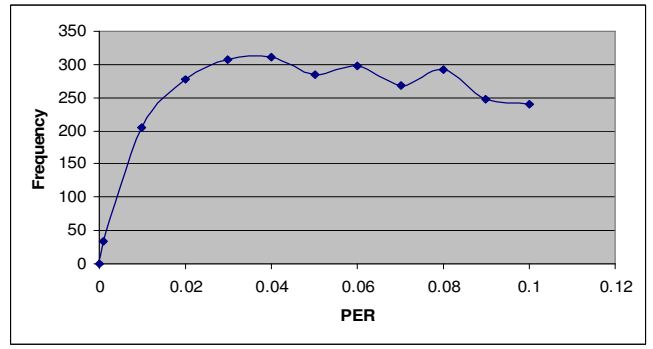

Fig. 34 Dup_ACK_Frequency

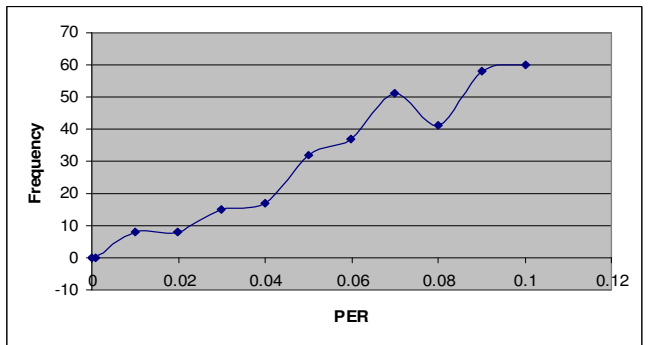

Fig. 35 Timeout_Frequency with PER

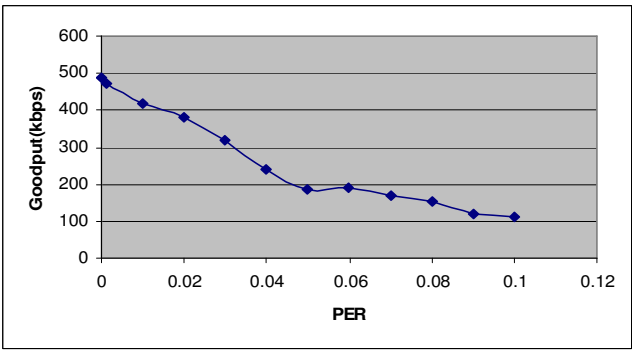

Fig. 36 Goodput (kbps) with PER

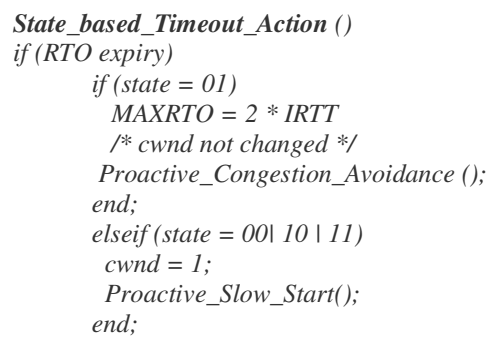

Fig. 37 State based Timeout Action

Fig. 33 shows the Frequency of three successive increases in mean RTT values within the simulation time which triggers a proactive correction of congestion window. As the PER increases this frequency is seen to reduce because with more errors the amount of data transmitted by the protocol also reduces. This frequency is important from the fact that with each such occurrence there is a proactive decrease of the congestion window [20]. Another important fact which is observed is that, at very high error rates when the amount of data transmitted is reduced there is an appreciable amount of proactive reduction attributed by error initiated RTT increase. Fig. 34 shows the frequency of DUP ACK reception with PER and it has been seen that initially the DUP ACK frequency increases with increasing PER and then it reduces since less data being transmitted because of frequent reduction of the congestion window during the DUP ACK handling. Fig. 35 shows the frequency of occurrence of timeout along with PER. It can be seen that as the PER increases the frequency of timeout increases appreciably, with no timeout for PER of $0.001,8$ timeouts for PER of 0.01 and 60 timeouts for PER of 0.1. The timeouts are the most expensive events in terms of retransmission and increase of RTO [18] value leads to the very high degradation [13] of throughput. Fig. 36 shows the achieved goodput [1] with varying PER of the individual connection. The maximum possible 
bandwidth is 500kbps and it can be seen that $488.5 \mathrm{kbps}$ is achieved at PER of 0.0001 but the throughput drastically falls to $111.2 \mathrm{kbps}$ at a PER of 0.1. From Fig. 32 to Fig. 36 the major factor which has to be considered in the design, for the performance improvement under high error condition has been analyzed so as to improve the performance of the protocol in high error cases. In Adaptive Multi-State Proactive TCP design the following new concepts have been used to enhance the performance.

\subsection{State based Timeout Action}

In Multi-State TCP a modification has been done and the congestion window will not be reduced to one in case of timeout event when the state of the network is 01 as shown in Fig. 37. In other states like 00,10 and 11 the normal timeout algorithm is used and with a timeout the congestion window is reduced to one and Proactive Slow Start [20] begins which prevents the protocol from a much degraded throughput in case of misclassification of states. Also in Multi State TCP we propose to use a modified logic for the calculation Maximum Retransmission Timeout [18]. Basically we want to keep the RTO [18] values within limit and in error induced loss the binary backoff algorithm leads to severe performance reduction. In Multi State TCP during state 01 i.e. when no congestion and high error dominates the network the Max RTO will be kept to twice the Ideal RTT value. An ns2 [12] based simulation has been carried out to find the optimal value of the maximum retransmission timeout so that the protocol gets an optimal throughput in all types of packet error rates. Fig. 38 shows the simulation result obtained by using different values of the MAXRTO. In the simulation for No Congestion and High Error state 01 this MAXRTO [18] value is used and other states used the normal MAXRTO of up to six binary backoff [18] leading to 64 times initial RTO. Since the protocol uses the normal Timeout mechanism for the other states only in very high PER of 0.1(-10db) an improvement can be seen when the MAXRTO is kept at twice the ideal RTT in Fig 38.

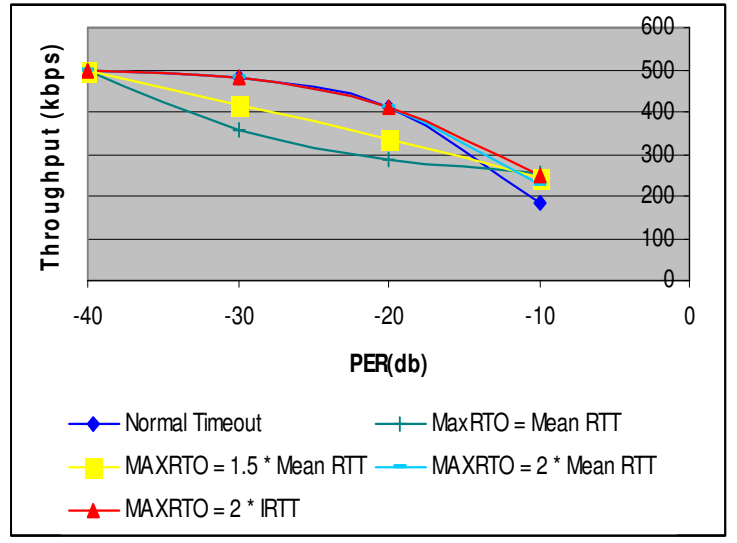

Fig. 38 Throughput vs. PER for values of MAXRTO

TABLE IV

SIMULATION RESULT FOR PROTOCOL

\begin{tabular}{|c|c|c|c|c|c|}
\hline PER & $\begin{array}{c}\text { Total } \\
\text { numbe } \\
\text { rof } \\
\text { Windo } \\
\text { ws }\end{array}$ & $\begin{array}{c}\text { RTT } \\
\text { Increa } \\
\text { se } \\
\text { Freq }\end{array}$ & $\begin{array}{l}\text { Dup } \\
\text { ACK } \\
\text { Freq }\end{array}$ & $\begin{array}{c}\text { Timeo } \\
\text { ut } \\
\text { Freq }\end{array}$ & $\begin{array}{l}\text { Throu } \\
\text { ghput } \\
\text { (kbps) }\end{array}$ \\
\hline $\begin{array}{l}.000 \\
1\end{array}$ & 842 & 179 & 0 & 0 & 488.5 \\
\hline .001 & 819 & 138 & 33 & 0 & 473.0 \\
\hline .01 & 675 & 70 & 204 & 8 & 419.4 \\
\hline .02 & 609 & 46 & 277 & 8 & 381.1 \\
\hline .03 & 566 & 46 & 307 & 15 & 318.8 \\
\hline .04 & 533 & 41 & 311 & 17 & 240.7 \\
\hline .05 & 480 & 53 & 285 & 32 & 185.0 \\
\hline .06 & 445 & 35 & 298 & 37 & 192.3 \\
\hline .07 & 381 & 41 & 269 & 51 & 170.3 \\
\hline .08 & 403 & 45 & 293 & 41 & 151.3 \\
\hline .09 & 353 & 41 & 248 & 58 & 118.4 \\
\hline .1 & 318 & 40 & 241 & 60 & 111.2 \\
\hline
\end{tabular}

\subsection{State based Error Recovery}

Multi State TCP uses a state based error handling mechanism. The major changes in Multi State TCP are in state 01 where no reduction of congestion window is done in case of DUP ACK reception. Only the Fast Retransmit is done of the lost packet. The logic is that once it is known that the lost packet is because of error and not congestion the reduction in congestion window will only degrade the performance [17][18]. For state 00 reduction of congestion window is kept to $3 / 4$ of its prevailing value instead to $1 / 2$. The logic is already a proactive action is being taken periodically with mean rise for three successive windows so reduction of $3 / 4$ instead of $1 / 2$ may enhance the performance especially when the state is estimated to have no congestion and error. 
In state 10 or 11 where the congestion is estimated to be present the reduction factor is kept at $1 / 2$ so that the fairness property [10][11] of the protocol is maintained under presence of congestion as shown in Fig. 39.

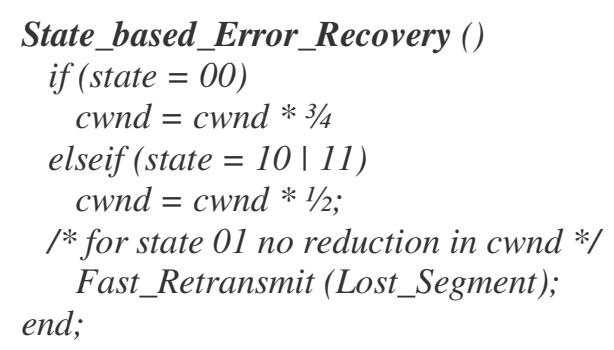

Fig. 39 State_based_Error_Recovery Algorithm

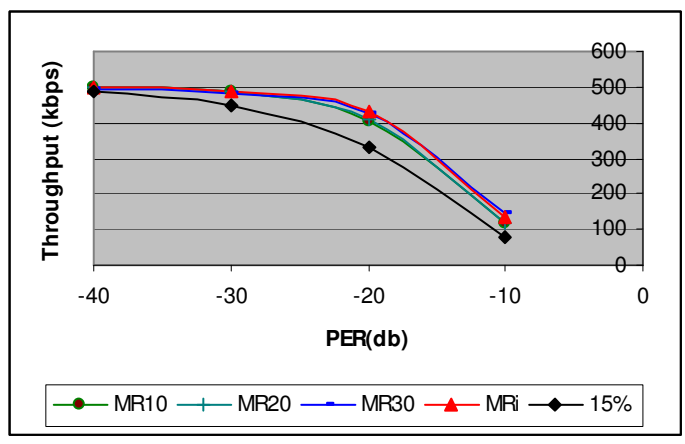

Fig. 40 Throughput vs. PER for different Penalty Factor

\subsection{Adaptive Penalty Factor}

There is a need to estimate the amount of extra data in the router buffer and to proactively adjust the congestion window to keep the extra data in the network within control [20]. The Mean Rise MRi calculated from all the RTT samples basically denotes the percentage by which the network buffers are overloaded. So $(1-\mathrm{MRi})$ can be considered to be a very good estimate of the penalty factor[20] which is multiplied with the congestion window on every three successive rise of Mean RTT. In Fig. 40 a comparison has been made to see the impact of the choice of penalty factor on the throughput of the protocol for different values of PER. It has been seen that use of the different Mean Rise values gives a better throughput than the flat 15\% [20] obtained empirically through a series of simulations in ns2 [12]. Out of all the Mean Rise values MRi gives the best throughput when used for the penalty factor considering all PER rates.

\subsection{State based Proactive Action}

At very high channel error condition the probability of an error induced increase of RTT is more than congestion induced increase of RTT [19] [23] [24]. So in state 01 there will be no proactive reduction of the congestion window with the penalty factor [20] so that the congestion window is not reduced to a very low value. Moreover it has also been seen that probability of state transition from state 11 to state 01 is more because high congestion high error state often leads to state 01 , as whenever there is more errors in the channel timeouts are inevitable and that leads to a drastic reduction of the congestion window and hence the overall transmission rate leading to the state01. So, high congestion high error network states are also taken care with the modifications made in this algorithm.

\section{TEST AND EVALUATION}

We evaluate the performance of the proposed TCP in terms of goodput through simulations when several connections share the same link. We simulate the system as shown in the Fig. 42 below where $\mathrm{N}$ senders transmit data to $\mathrm{N}$ receivers through a satellite channel [1]. The $\mathrm{N}$ streams are multiplexed in Earth Station A, whose buffer can accommodate K segments. The segments may get lost with a packet error rate PER. In this experiment all the $\mathrm{N}$ senders are each connected to the Earth station A with a link of bandwidth 500kbps. All the $\mathrm{N}$ receivers are connected to Earth station B with a 500kbps link. We have taken $\mathrm{N}=10, \mathrm{~K}=25$ segments, receiver window rwnd $=64$ segments, the link between Earth Station A to B via satellite to be $5 \mathrm{Mb}$ and the RTT between the two stations as 550ms. All the results in this section have been obtained by considering the system behavior for T_Simulation $=550 \mathrm{~s}$ which is 1000 times the round trip time value. 


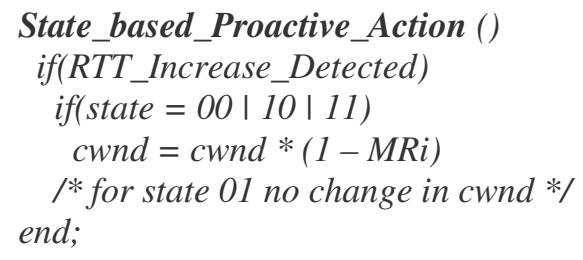

Fig. 41 State based Proactive Action Algorithm

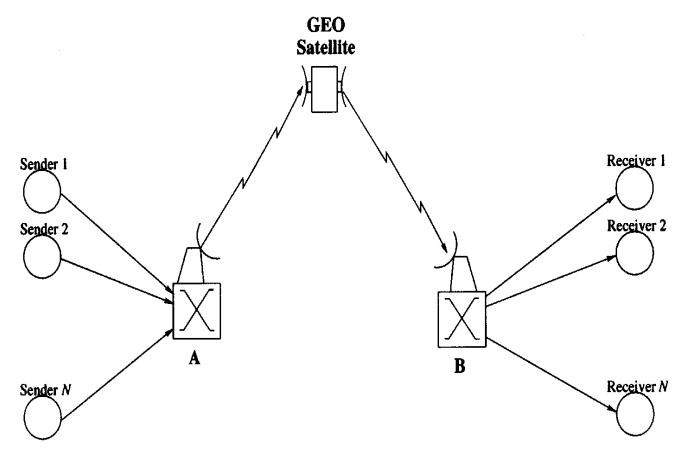

Fig. 42 Simulation Scenario

\subsection{Goodput Calculation}

Goodput is the effective amount of data delivered through the network [1]. It is a direct indicator of network performance. We expect that a good TCP scheme transmit as much data as possible, while behaving friendly to other TCP flows in terms of consuming the network resource. In the following graphs shown the throughput of the protocol is compared to TCP SACK [6] and TCP Vegas [5] in the same test bed of 10 senders communicating to 10 receivers using a $5 \mathrm{Mb}$ bottleneck link via satellite. To artificially induce congestion the bandwidth between Earth Station A and B is stepwise reduced to see the reaction of the protocol to congestion. Here all ten different connections are connected to the Earth Station with 500kbps bandwidth each. So the minimum aggregate bandwidth required for all the connections to perform optimally is 5Mbps. Table V shows the goodput of the Multi State TCP along with Vegas [5], TCP SACK [6] and Proactive TCP [20]. In Table VI the percentage utilization is shown and it can be seen that Multi State TCP utilizes 50\% bandwidth even in a very high PER of 0.1 . Table VII shows the appreciation over its peers and in high error condition the appreciation is $600 \%$ over Vegas and $800 \%$ over SACK. This is a very significant improvement achieved with following graphs showing the result in Fig. 43, Fig. 44 and Fig. 45.

\begin{tabular}{ccccc}
\multicolumn{7}{c}{ TABLE V } \\
GOODPUT FOR CONGESTED NETWORK WITH PER \\
\hline PER & $\begin{array}{c}\text { Vegas } \\
(\mathrm{kbps})\end{array}$ & $\begin{array}{c}\text { SACK } \\
(\mathrm{kbps})\end{array}$ & $\begin{array}{c}\text { Proactive } \\
\text { TCP } \\
(\mathrm{kbps})\end{array}$ & $\begin{array}{c}\text { MultiState } \\
\text { TCP } \\
(\mathrm{kbps})\end{array}$ \\
\hline 0.001 & 244.6 & 225 & 450 & 481 \\
\hline 0.01 & 168.7 & 116 & 323.6 & 408.5 \\
\hline 0.1 & 34.9 & 27 & 86.67 & 244.9 \\
\hline
\end{tabular}

TABLE VI

\begin{tabular}{ccccc}
\multicolumn{6}{c}{ PERCENTAGE BANDWIDTH UTILIZATION } \\
\hline PER & $\begin{array}{c}\text { Vegas } \\
(\%)\end{array}$ & $\begin{array}{c}\text { SACK } \\
(\%)\end{array}$ & $\begin{array}{c}\text { Proactive } \\
\text { TCP }(\%)\end{array}$ & $\begin{array}{c}\text { MultiState } \\
\text { TCP }(\%)\end{array}$ \\
\hline 0.001 & 48.92 & 45.00 & 90.00 & 96.20 \\
\hline 0.01 & 33.74 & 23.20 & 64.7 & 81.70 \\
\hline 0.1 & 6.98 & 5.40 & 17.33 & 48.99 \\
\hline
\end{tabular}

TABLE VIII

TABLE VII PERCENTAGE APPRECIATION OF MULTI-STATE

\begin{tabular}{cccc}
\multicolumn{4}{c}{ TCP OVER ITS PEERS FOR DIFFERENT PER } \\
\hline PER & Vegas (\%) & SACK $(\%)$ & $\begin{array}{c}\text { Proactive } \\
\text { TCP }(\%)\end{array}$ \\
\hline 0.001 & 96.64 & 113.77 & 6.88 \\
\hline 0.01 & 142.14 & 252.15 & 26.23 \\
\hline $\mathbf{0 . 1}$ & $\mathbf{6 0 1 . 9 7}$ & $\mathbf{8 0 7 . 3 7}$ & $\mathbf{1 8 2 . 6 6}$ \\
\hline
\end{tabular}

Jain's Fairness Index for different Packet Error rates

\begin{tabular}{ccccc}
\multicolumn{5}{c}{ Jain's Fairness Index for different Packet Error rates } \\
\hline 000 & 0.0001 & 0.001 & 0.01 & 0.1 \\
\hline 0.99999 & 0.999684 & 0.999415 & 0.999626 & 0.998864 \\
\hline
\end{tabular}

In Table VIII the Jain's fairness index [11] calculated from all the 10 connections for different packet error rate is shown. It can be seen that all the connections get an almost equal share of bandwidth [10] with a very slow degradation of fairness with increasing PER. 


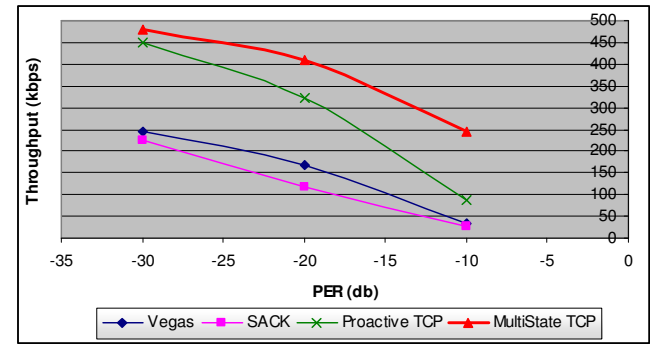

Fig. 43 Throughput for Congested Network with different PER

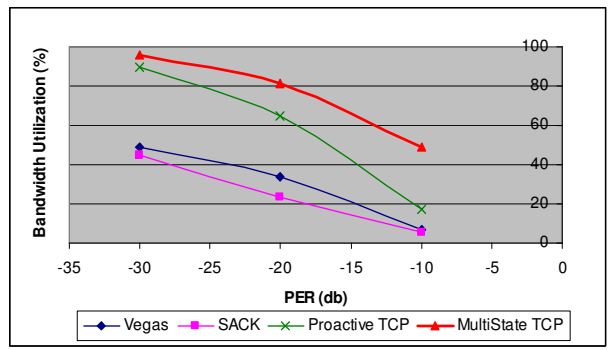

Fig. 44 Percentage Bandwidth Utilization

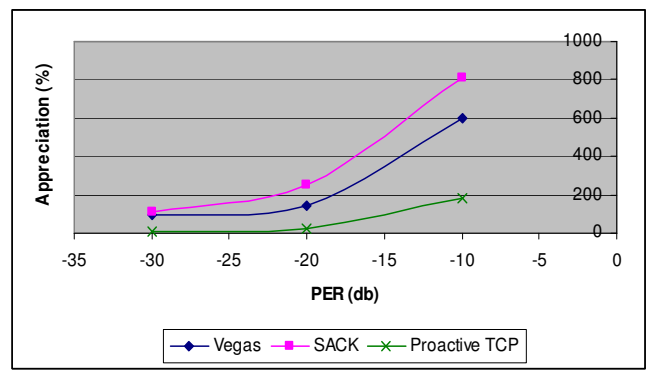

Fig. 45 Multi-State TCP Appreciation in percentage compared to its peers for different PER

\section{CONCLUSION}

This paper through detailed simulation and analysis of TCP over Satellite Networks has established the fact that multi state network classification is essential to enhance the overall throughput of the network which is affected equally by congestion and channel noise. The modelling and analysis as discussed in this paper has proved that the conventional two state network classification devised in classical TCP over wired network is not optimal for satellite based TCP protocol. So to enhance the performance of TCP protocols over Satellite based Networks there is a need for a Multi State representation of network condition by a combination of the Multi State Network Classification model to be developed which has been explained in this paper. For the design of such a model simulation based experiments are carried out to analyze the impact of congestion and error on RTT values. The inferences drawn from the analysis have been used to evolve a novel Statistical RTT based model which uses a hierarchical decision making process for network state classification. This model has been utilized in the design of an Adaptive Multi State Proactive Transport Protocol for performance enhancement of satellite based networks. The major causes of performance degradation have been analyzed and the novel schemes for throughput enhancement have been proposed. The design consideration of the protocol has been discussed thoroughly in this paper and it has been shown how an adaptive mechanism of learning the statistical parameters of a network can help in getting a significant performance benefit. The Multi-State TCP is seen to outperform substantially over its peers mainly in high error conditions. The most important merit is that the performance improvement obtained in this protocol [14] does not need any change in the Routers and all changes are restricted to the sender and receiver protocol stack [7].

\section{Acknowledgements}

Our special thanks to Mr. V.S.Palsule, Deputy Director, Satellite Navigation Application Area, SAC, ISRO, Mr.A.P.Shukla, Head, ACTD and Mr. N.G.Vasantha Kumar, Senior Scientist, ACTD for providing constant encouragement towards the realization of this work.

\section{References}

[1] Ian F. Akyildiz, Giacomo Morabito, Sergio Palazzo, (2001) “TCP-Peach: A New Congestion Control Scheme for Satellite IP Networks”, IEEE/ACM TRANSACTIONS ON NETWORKING, VOL. 9, NO. 3, JUNE 
[2] Ian F. Akyildiz, Xin Zhang, Jian Fang, (2002) "TCP-Peach+: Enhancement of TCP-Peach for Satellite IP Networks", IEEE COMMUNICATIONS LETTERS, VOL. 6, NO. 7, JULY

[3] Ozgur B. Akan, Jian Fang, Ian F. Akyildiz, (2004) “TP-Planet: A Reliable Transport Protocol for InterPlaNetary Internet”, IEEE/SAC, vol 22, no 2, Feb. 2004, pp 348-61

[4] Jian Fang and Özgür B. Akan,(2004)"Performance of Multimedia Rate Control Protocols in InterPlaNetary Internet", IEEE COMMUNICATIONS LETTERS, VOL. 8, NO. 8, AUGUST

[5] L. S. Brakmo, S. O Malley, L. L. Peterson, (1994) “TCP Vegas: New Techniques for Congestion Detection and Avoidance”, Proc. ACM SIGCOMM 1994, pp. 24-35, October

[6] Mathis, M., J. Mahdavi, S.Floyd, and A.Romanow, (1996) "TCP Selective Acknowledgment Options", RFC 2018, April

[7] J. Martin, A. Nilsson, and I. Rhee, (2003) "Delay-Based Congestion Avoidance for TCP", IEEE/ACM Transactions on Networking, vol. 11, no. 3, pp. 356-369, June

[8] M. Mathis, J. Mahdavi, (1996) "Forward Acknowledgment: Refining TCP Congestion Control", Proc. ACM SIGCOMM 1996, pp. 281-292, Aug.

[9] J. Padhye, V. Firoio, D. Towsley, J. Kurose, (2000) "Modeling TCP Reno Performance: A Simple Model and Its Empirical Validation”, IEEE/ACM Trans. Networking, Vol. 8, No. 2, pp. 133-145, April

[10] S.C. Tsao, Y.C. Lai, and Y.D. Lin, (2007) "Taxonomy and Evaluation of TCP-Friendly Congestion-Control Schemes on Fairness, Aggressiveness, and Responsiveness", IEEE Network, November

[11] R. Jain, D. Chiu, and W. Hawed, (1984) "A quantitative measure of fairness and discrimination for resource allocation in shared computer systems", DEC, Res. Rep.TR-301,

[12] UCB/LBNL/VINT Network Simulator [Online]. Available: http://www.isi.edu/nsnam/ns/

[13] T.V.Lakshman and U.Madhow, (1997) "The performance of TCP/IP for networks with high bandwidth-delay products and random loss", IEEE/ACM Trans. Networking, vol. 5, June

[14] Injong Rhee and Lisong Xu, (2007) "Limitations of Equation-based Congestion Control”, IEEE/ACM Trans. Networking, June

[15] C. Metz, (1999) "TCP over satellite the final frontier", IEEE Internet Compuingting., pp. 76-80, Jan. /Feb

[16] C. Partridge and T. J. Shepard, (1997) “TCP/IP performance over satellite links", IEEE Network Magazine., pp. 44-49, Sept-Oct.

[17] W. Stevens, (1994) “TCP/IP Illustrated” Addison-Wesley, vol.1

[18] V. Jacobson, (1988) "Congestion avoidance and control," in Proc. ACM SIGCOMM

[19] Ravi.S.Prasad, "On the Effectiveness of Delay-Based Congestion Avoidance “

[20] Mohanchur Sarkar, K.K.Shukla, K.S.Dasgupta, (2010) "A Proactive Transport Protocol for Performance Enhancement of Satellite based Networks" in (IJCA) International Journal of Computer Applications, Vol 1, Number16, pp. 114-121, Feb

[21] M. Allman, (2000), “Ongoing TCP research related to satellites”, RFC 2760, Feb.

[22] Jiang Wu, Hosam El-Ocla , (2004) "TCP Congestion Avoidance Model with Congestive Loss", in Proceedings $12^{\text {th }}$ IEEE International Conference, 2004, Vol 1 , pp. 3-8

[23] Abhishek Murthy, (2006) "Correlation Coefficient based Loss Differentiation Algorithm (CCLDA) for TCP vis-à-vis traditional LDAs", 4th Conference on Research and Development

[24] S. Biaz, (2003) "Is the round-trip time correlated ... flight" Internet Measurement Conference 